Purdue University

Purdue e-Pubs

2017

\title{
Axisymmetric Wall Jet Development in Confined Jet Impingement
}

T. Guo

M. Rau

P. Vlachos

S V. Garimella

Purdue Univ, sureshg@purdue.edu

Follow this and additional works at: http://docs.lib.purdue.edu/coolingpubs

Guo, T.; Rau, M.; Vlachos, P.; and Garimella, S V., "Axisymmetric Wall Jet Development in Confined Jet Impingement" (2017). CTRC Research Publications. Paper 316.

http://dx.doi.org///dx.doi.org/10.1063/1.4975394

This document has been made available through Purdue e-Pubs, a service of the Purdue University Libraries. Please contact epubs@purdue.edu for additional information. 


\title{
Axisymmetric wall jet development in confined jet impingement
}

\author{
Tianqi Guo, Matthew J. Rau, Pavlos P. Vlachos, ${ }^{\text {a) }}$ and Suresh V. Garimella \\ School of Mechanical Engineering, Purdue University, 585 Purdue Mall, West Lafayette, Indiana 47907, USA
}

(Received 20 August 2016; accepted 20 January 2017; published online 15 February 2017)

\begin{abstract}
The flow field surrounding an axisymmetric, confined, impinging jet was investigated with a focus on the early development of the triple-layered wall jet structure. Experiments were conducted using stereo particle image velocimetry at three different confinement gap heights $(2,4$, and 8 jet diameters) across Reynolds numbers ranging from 1000 to 9000 . The rotating flow structures within the confinement region and their interaction with the surrounding flow were dependent on the confinement gap height and Reynolds number. The recirculation core shifted downstream as the Reynolds number increased. For the smallest confinement gap height investigated, the strong recirculation caused a disruption of the wall jet development. The radial position of the recirculation core observed at this small gap height was found to coincide with the location where the maximum wall jet velocity had decayed to $15 \%$ of the impinging jet exit velocity. After this point, the self-similarity hypothesis failed to predict the evolution of the wall jet further downstream. A reduction in confinement gap height increased the growth rates of the wall jet thickness but did not affect the decay rate of the wall jet maximum velocity. For jet Reynolds numbers above 2500, the decay rate of the maximum velocity in the developing region of the wall jet was approximately -1.1 , which is close to previous results reported for the fully developed region of radial wall jets. A much higher decay rate of -1.5 was found for the wall jet formed by a laminar impinging jet at $\mathrm{Re}=1000$. Published by AIP Publishing. [http://dx.doi.org/10.1063/1.4975394]
\end{abstract}

\section{INTRODUCTION}

Impinging jets are ubiquitous in engineering applications. Jets are used for cooling the leading edge of gas turbines, ${ }^{1}$ rapid processing of foods, ${ }^{2}$ quenching of metal parts, ${ }^{3}$ and thermal management of electronic components. ${ }^{4}$ When used for cooling, impinging jets can achieve high heat transfer rates compared with other common single-phase cooling methods such as forced convection parallel to a surface or natural convection. ${ }^{5}$ Confined impinging jets, in particular, are of interest for compact electronics packaging. ${ }^{6}$

The flow field resulting from jet impingement consists of several regions with distinct flow features, ${ }^{7}$ as shown in Fig. 1(a). A free jet region forms as fluid exits the orifice in the confinement plate. The jet potential/inviscid core, where the velocity is relatively uniform, is surrounded by a free shear layer where the jet entrains the ambient fluid and slowly expands. ${ }^{8}$ As the jet approaches the bottom plate, the flow decelerates in the vertical direction and accelerates in the radial direction near the stagnation point. This acceleration in the radial direction forms a wall jet along the bottom wall of the confinement gap.

Wall jets are characterized by a triple-layered structure, as shown in Fig. 1(b). The first detailed study of a wall jet offered theoretical boundary layer solutions for a jet spreading over a planar surface. ${ }^{9}$ Wall jets have been shown to display incomplete self-similarity, where the inner and outer regions

\footnotetext{
a) Author to whom correspondence should be addressed. Electronic mail: pvlachos@purdue.edu
}

of the flow scale differently as described by the triple-layer hypothesis for planar wall jets. ${ }^{10}$ Incomplete self-similarity requires that the maximum velocity $\left(u_{m}\right)$ and wall jet halfwidth $\left(Y_{1 / 2, i}\right)$ be functions of the radial downstream location $(x / d$, where $d$ is the jet orifice diameter) in the forms

$$
\frac{u_{m}}{V_{j}} \propto\left(\frac{x}{d}\right)^{\alpha}
$$

and

$$
\frac{Y_{1 / 2, i}}{d} \propto\left(\frac{x}{d}\right)^{\beta_{i}}
$$

$V_{j}$ corresponds to the jet exit velocity. The exponent $\alpha$ is referred to as the decay rate of $u_{m}$, and $\beta_{i}$ is the growth rate of $Y_{1 / 2, i}$, where $i=T, W$ indicates the top layer or wall layer, respectively. To better perform scaling analysis, a nominal jet half-width $\left(y_{1 / 2, i}\right)$ is introduced and given by a linear fit to experimental data plotted on a bi-logarithmic scale.

In the case of a confined impinging jet, a recirculation region also exists in which the flow is driven by both the impinging jet and the wall jet. ${ }^{11,12}$ The flow rotates at a lower velocity than the vertical jet and wall jet regions, forming stable toroidal vortex structures as depicted in Fig. 1(a). This recirculation of fluid has been shown to enhance the heat exchange process in the radial distances between $x / d=1.5$ and $4 .{ }^{13}$

Although the flow field created by an impinging jet has attracted numerous research efforts for both heat transfer and fluid dynamics applications, the wall jet development in a confined geometry has received little attention. The current study undertakes a detailed experimental investigation of the influence of confinement on the flow field and radial wall jet 


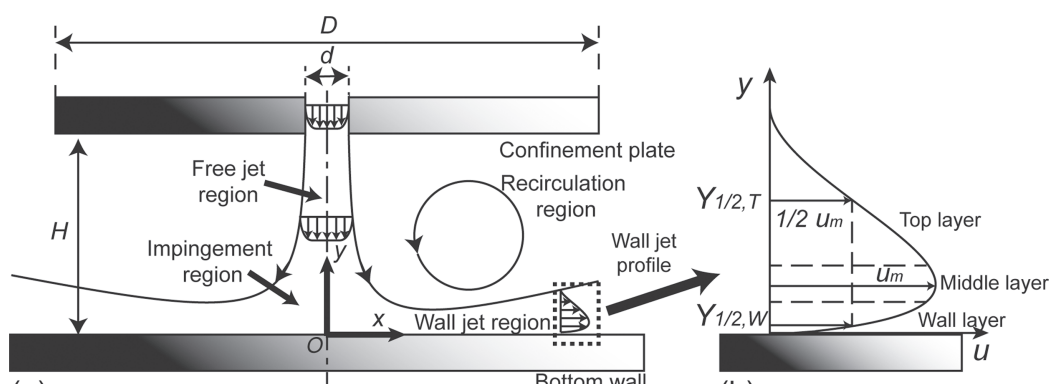

(a) Bottom wall (b)
FIG. 1. Schematic drawings of (a) the axisymmetric flow field formed by the confined impinging jet and (b) the triple-layered wall jet structure. development. Special attention is given to the effect of the recirculation pattern on the early development of the wall jet, and the notion of incomplete self-similarity is explored. To the best of the authors' knowledge, this is the first experimental study of the effect of confinement on the self-similar structure of a radial wall jet.

\section{EXPERIMENTAL METHOD}

\section{A. Test section}

A detailed description of the experimental facility was provided by Rau and Garimella. ${ }^{14}$ Only salient details of the facility and the stereo particle image velocimetry (SPIV) measurement system are included here. A schematic drawing of the test section is shown in Fig. 2. The working fluid was deionized water, which was circulated through a closed flow loop by a gear pump. The experiments were conducted at room temperature. The test section chamber (width $\times$ length $\times$ height $=132 \mathrm{~mm} \times 132 \mathrm{~mm} \times 100 \mathrm{~mm}$ ) was large enough such that the flow in the confined region remained unaffected by the flow exiting the test section. The jet was issued from a round, sharp-edged orifice $3.75 \mathrm{~mm}$ in diameter $(d)$ and two diameters in length. The orifice was located at the center of the upper confinement plate. Precision-machined stainless steel spacer pins defined the confinement gap height $(H)$ between the upper plate and the bottom wall, which may also be referred to as the orifice-to-plate distance. A $25.4 \mathrm{~mm}$-diameter $(6.7 d)$ circular copper surface was flush-mounted on the bottom wall of the confinement gap with its center aligned with the jet axis. This experimental arrangement allowed for heat transfer experiments in prior studies; ${ }^{15,16}$ however, no heat input was used in the current study. Due to a tiny gap around the perimeter of the copper surface (on the order of $100 \mu \mathrm{m}$ ), a small flow disruption was introduced in the wall layer of the wall jet at this location. The analysis and interpretation of the results in the wall layer region reflect this experimental artifact. The diameter $(D)$ of the upper confinement plate was $70 \mathrm{~mm}$, resulting in a confinement gap that extended 9.3 jet diameters downstream from the jet axis.

Two high-speed cameras (Phantom Miro M340, Vision Research) were installed with viewing angles of $35^{\circ}$ and $10^{\circ}$ relative to the light-sheet normal direction, as shown in Fig. 2. Lens tilt adapters were used to satisfy the Scheimpflug condition for SPIV. ${ }^{17}$ The polycarbonate front and back walls of the test section were transparent and allowed full optical access; the side walls were made from opaque polyether-ether ketone (PEEK). A $2 \mathrm{~mm}$-thick laser sheet was introduced into the test section through the back wall and reflected across the axisymmetric plane of interest with a mirror mounted inside the test section, as shown in Fig. 2. The axisymmetry of the flow field was confirmed by a special test case with one camera looking

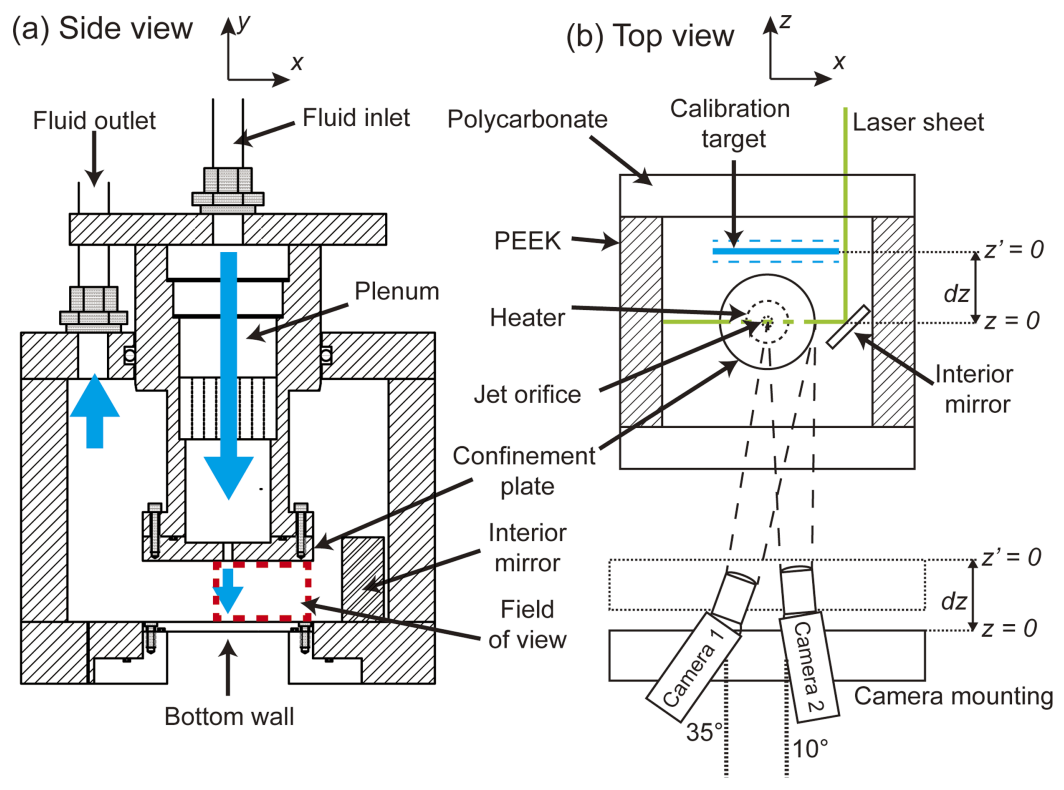

FIG. 2. A schematic drawing showing the (a) side view and (b) top view of the test section and camera positions for data recording and camera calibration. 
at the entire confinement gap at $H / d=4$. The thickness of the laser sheet was designed to capture the maximum out-ofplane fluctuation velocity $\left(w^{\prime}\right)$ due to vortex shedding from the jet orifice. Bias errors in the radial velocity measurements resulting from of the light sheet thickness and axisymmetric flow were found to be negligible for $x / d>1$. Thus, wall jet results are only presented in this range.

\section{B. Data recording}

The jet exit velocity $\left(V_{j}\right)$ and the time delay $(\Delta t)$ between laser pulses are listed in Table I. The liquid flow rate was obtained by an in-line Coriolis flow meter (CMFS015M, Emerson) and used to calculate the averaged jet exit velocity $\left(V_{j}\right)$. Five Reynolds numbers $\left(\operatorname{Re}=V_{j} d / v\right.$, where $v$ is the kinematic viscosity) ranging from 1000 to 9000 at confinement heights of $H / d=2,4$, and 8 were investigated. The double pulses from the laser (Nd:YLF Terra PIV 527-80-M, Continuum, $527 \mathrm{~nm}$ ) were synchronized with the cameras recording single-image exposures at an image-pair frequency of $750 \mathrm{~Hz}$ by a precision multi-channel delay generator (TSI Laserpulse Synchronizer 610036). One thousand image pairs over $1.33 \mathrm{~s}$ were recorded for each test case, and the recording time interval was long enough to obtain meaningful averaged measurements.

Fluorescent polystyrene microspheres of $10 \mu \mathrm{m}$ diameter (Spherotech) were used as seeding particles. The seeding density was maintained at a minimum of 10 particles per $32 \times 32$ pixel window by periodic re-seeding. This ensured sufficient mutual information between sequential images for meaningful cross correlation with low measurement uncertainty. ${ }^{18,19}$

\section{Camera calibration}

Camera calibrations, mapping image coordinates to world coordinates, were obtained by calibrating each camera against a single-layer transparent glass target (FA131, Max Levy Autograph). The calibration followed the 3D procedure outlined by Prasad. ${ }^{20}$ For calibration, the cameras and the target were translated a distance $(d z)$ from the axisymmetric plane of interest so that the calibration target could move without obstruction from the inner mirror, as shown in Fig. 2. Calibration images recorded at planes from $z^{\prime}=-1.5 \mathrm{~mm}$ to $z^{\prime}$ $=1.5 \mathrm{~mm}$ in $0.5 \mathrm{~mm}$ increments were used to construct the calibration mapping functions. Self-calibration was then performed using particle images to correct the disparities between two cameras. ${ }^{21}$ The final mapping functions were applied in the three-component velocity reconstruction using the twodimensional, two-component vector field from each camera based on the generalized reconstruction procedure. ${ }^{22}$

TABLE I. Test matrix with jet velocity and time delay between laser pulses.

\begin{tabular}{lccc}
\hline \hline $\operatorname{Re}$ & $H / d$ & $V_{j}(\mathrm{~m} / \mathrm{s})$ & $\Delta t(\mu \mathrm{s})$ \\
\hline 1000 & $2,4,8$ & 0.24 & 1000 \\
2500 & $2,4,8$ & 0.60 & 460 \\
5000 & $2,4,8$ & 1.21 & 350 \\
7500 & $2,4,8$ & 1.79 & 220 \\
9000 & $2,4,8$ & 2.08 & 180 \\
\hline \hline
\end{tabular}

\section{Data processing}

Prior to PIV evaluations, the images were pre-processed to eliminate background noise. A local minimum subtraction was applied at each pixel across the entire time series for each test case. An in-house PIV code, Prana (https://github.com/aetherlab/prana) implemented in MATLAB (Mathworks) was used to perform the PIV evaluation and stereo reconstruction. Cross correlation between frames was performed using the robust phase correlation (RPC). RPC substantially reduces bias errors and peak-locking effects in the presence of high shear and rotational motion in comparison with standard cross correlation algorithms. ${ }^{23-25}$ In addition, the time-averaged velocity field was obtained with an ensemble correlation scheme, ${ }^{26}$ which averages the 1000 correlation planes from 1000 image pairs. It has been shown that ensemble correlation is able to deliver more accurate time-averaged results compared to simply averaging the instantaneous measurements. ${ }^{27}$

For the overall flow field velocity evaluation, a $64 \times 64$ $(x \times y)$ pixel square-windowed interrogation region was used for the first pass and reduced to a $32 \times 32$ pixel window for the subsequent passes. A $75 \%$ window overlap delivered a final spatial vector resolution of $200 \mu \mathrm{m}$ in the $x$ and $y$ directions. A different set of PIV evaluation parameters was used to resolve the thin wall jet. After the initial pass with a $64 \times 64$ pixel window, a window size of $64 \times 4$ pixels was used for a spatial resolution of $25 \mu \mathrm{m}$ in the $y$ direction. A $75 \%$ window overlap was used to better resolve the velocity gradient in the shear region, ${ }^{28}$ and the narrow window size was enabled by using the ensemble correlation to increase the correlation strength, similar to the approach used by Westerweel et al. ${ }^{29}$

For all PIV evaluations, a continuous window offset with iterative window deformation ${ }^{30}$ was applied to compensate for in-plane velocity gradients. Each image in the entire image set was deformed according to the ensemble averaged velocity field after each pass, which was iteratively determined using a convergence criterion of $1 \%$ of the RMS difference between successive iterations. The first and intermediate passes were followed by vector validation, using universal outlier detection $(\mathrm{UOD})^{31}$ to remove and replace spurious vectors, and a Gaussian smoothing filter $(3 \times 3$ kernel with a standard deviation of 2). No smoothing was applied after the final pass.

\section{RESULTS}

\section{A. Overall flow pattern and recirculation structure}

Representative streamtraces from the ensemble-averaged flow field ( $x$ and $y$ resolution $200 \mu \mathrm{m} \times 200 \mu \mathrm{m}$ ) overlaid on contours of normalized velocity magnitude $(|V|)$ are shown in Fig. 3. The typical values for the averaged and RMS of $w$ component are less than $1 \%$ and $10 \%$ of the jet exit velocity for the averaged flow field, and therefore negligible for most of the analysis in this section. In-plane velocity vectors are plotted in the regions of the impinging jet and the wall jet. The streamtraces indicate the presence of a toroidal recirculating structure. At confinement gap heights of $H / d=2$ (at $\operatorname{Re}=1000$ and 9000) and $H / d=4($ at $\operatorname{Re}=1000)$, a counterclockwise recirculation is observed in agreement with the laser Doppler velocimetry (LDV) results reported by Fitzgerald and 


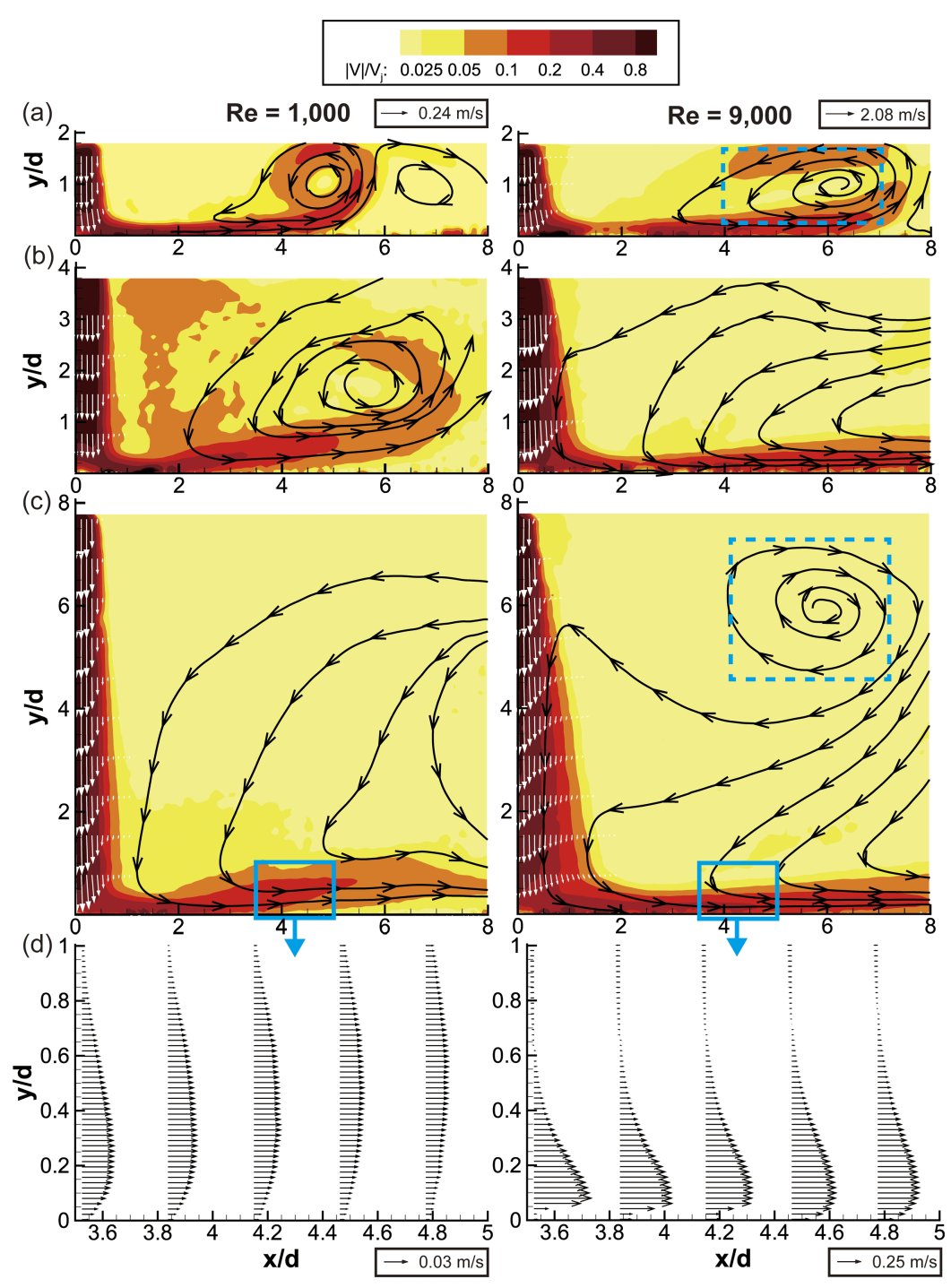

FIG. 3. Streamtraces overlaid on contours of velocity magnitude for $\operatorname{Re}=1000$ in the left column and $\mathrm{Re}=9000$ in the right column, at confinement heights $H / d$ of (a) 2, (b) 4, and, (c) $8 . U$ velocity component is plotted within the wall jet regions in the range indicated by the solid-line windows for $\mathrm{Re}=1000$ and $\mathrm{Re}=9000$ at $H / d=8$ in (d). The dashed windows show the spatial locations plotted in Fig. 4.

Garimella. ${ }^{12}$ This toroidal vortex causes liquid to recirculate from the wall jet back towards the vertical impinging jet. In contrast, the vortex that is shown for $H / d=8$ at $\operatorname{Re}=9000$ in Fig. 3 rotates in a clockwise direction and is located away from the bottom wall near the top edge of the confinement gap. Representative wall jet velocity profiles are plotted in Fig. 3(d) for $\operatorname{Re}=1000$ and 9000 at $H / d=8$. The vectors are skipped in both the $x$ and $y$ directions, and only 1 out from 3 is shown for better presentation.

Fitzgerald and Garimella ${ }^{12}$ found that the counterclockwise recirculation present in their confined flow moved radially outwards in the confinement gap with increasing Reynolds number and with increasing gap heights for $H / d=3$ and $H / d=4$. Similar structures have also been found in numerical simulations without sidewalls. ${ }^{32,33}$ The results presented in Fig. 3 show a similar trend. At $H / d=2$, the center of the vortex is located at approximately $x / d=4.75$ at $\operatorname{Re}=1000$ and moves to approximately $x / d=6.25$ at $\mathrm{Re}=9000$. For increasing confinement gap height at $\mathrm{Re}=1000$, the vortex moves from $x / d=4.75$ at $H / d=2$ to $x / d=5.75$ at $H / d$ $=4$. A further shift downstream in vortex position with increasing gap height would cause the recirculation to be located outside of the measurement domain at $H / d=8$, which explains the absence of a counter-clockwise vortex in the results shown at $H / d=8$. Similar behavior is likely present with increasing gap height at $\mathrm{Re}=9000$, as the counter-clockwise vortex located at $x / d=6.25$ at $H / d=2$ would move out of the measurement domain at $H / d=4$ and $H / d=8$ at this Reynolds number. The streamtraces shown for $H / d=8$ at $\mathrm{Re}=9000$ are distinct due to the presence of a clockwise vortex. As will be discussed later in this work, no evidence is found regarding the effect of this clockwise vortex on the development of the wall jet.

Time-averaged vortex structures are identified using the criterion proposed by Zhou et al ${ }^{34}$ where the imaginary part of the complex eigenvalue of the velocity gradient tensor $\left(\lambda_{c i}\right)$ is calculated and referred to as the local swirling strength of the vortex. The centroids of the vortices are calculated and referred to as recirculation cores. Contours of the swirling strength are plotted in Fig. 4 for the $x$ and $y$ ranges shown with the dashed windows in Fig. 3. Note that the vertical range of the plot for the $H / d=8$ case is different from the $H / d=2$ case; although the same scaling is maintained in all plots. As shown in Figs. 4(a) -4 (c) for $H / d=2$, the outward movement of the recirculation structure is accompanied by a decrease in the magnitude of the swirling strength. Fig. 4(d) shows the vortex 

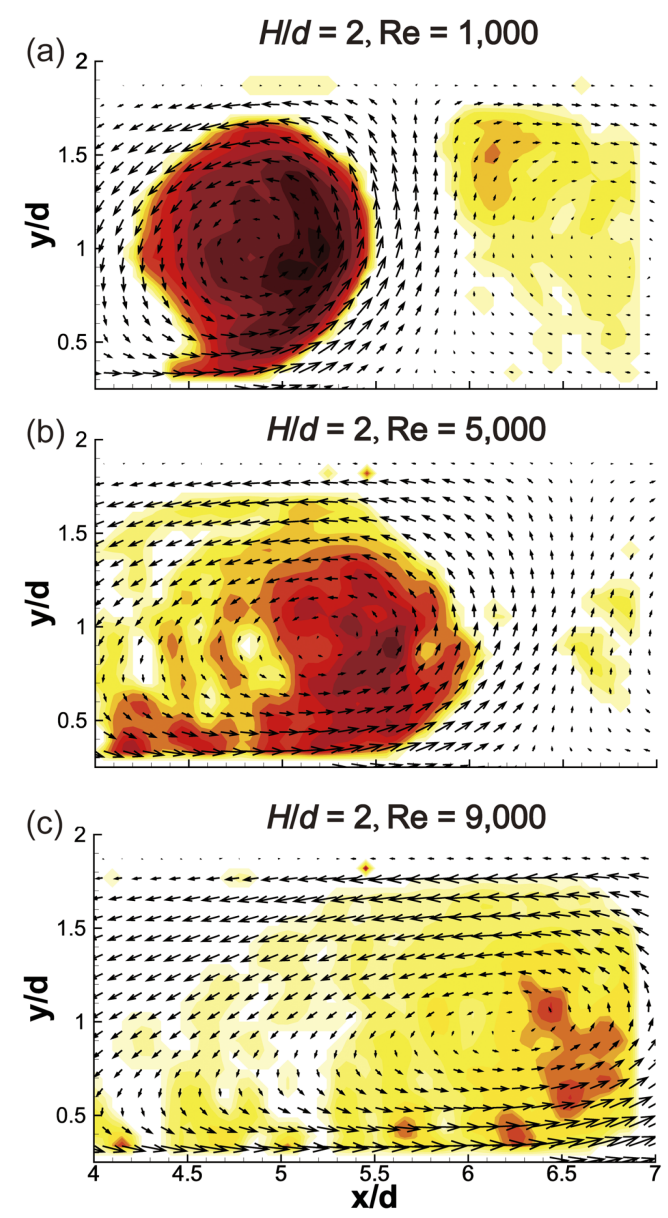
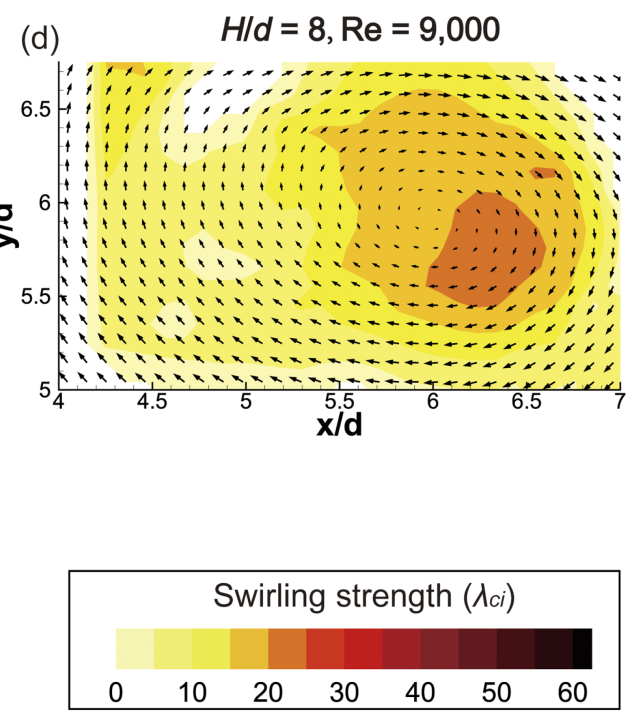

FIG. 4. The recirculation pattern and contours of the swirling strength plotted for the regions shown with the dashed rectangles in Fig. 3.

that occurs at $H / d=8$, which rotates weakly in the clockwise direction.

To quantify the conditions at the start of the wall jet formation, characteristics of the vertical jet before impingement are explored. The jet core length, jet expansion angle, and mass entrainment coefficient were obtained using the ensembleaveraged flow field, while the turbulent kinetic energy was calculated from the instantaneous flow field. Fig. 5 shows the jet core length $\left(l_{c}\right)$, defined as the distance from the jet orifice to the point where the centerline velocity has decayed to $95 \%$ of $V_{j},{ }^{35}$ and the jet expansion angle $(\theta)$ between the jet centerline direction and the jet boundary. The jet boundary was defined as the radial location where the vertical velocity has decreased to $5 \%$ of the centerline velocity $\left(v_{c}\right)$.

Fig. 6(a) shows $l_{c}$ non-dimensionalized by the jet orifice diameter for all test cases; the total confinement gap height for each case is shown with the color-coded dashed lines. The distance between the symbols and corresponding lines for each case is indicative of the distance between the impingement surface and the end of the jet core. For the least confined case of $H / d=8$, at $\operatorname{Re}=1000$ the jet core extends to almost $85 \%$ of the gap height before the jet impinges on the bottom plate, while at Reynolds numbers above 5000, the jet core length reduces to about four jet diameters (50\% of the gap height). As the upper confinement plate moves closer to the bottom plate, the distance between the end of the jet core and the bottom plate gets smaller. This distance is at a minimum (about (a)

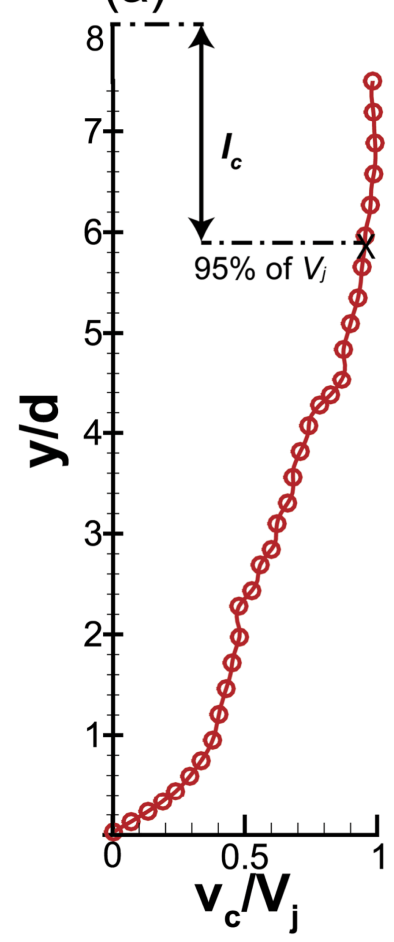

(b)

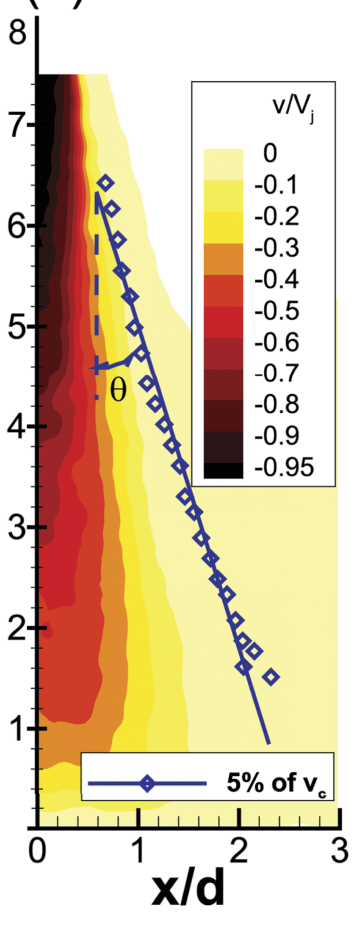

FIG. 5. Example results showing the determination of (a) the jet core length $\left(l_{c}\right)$ and (b) jet expansion angle $(\theta)$ for the impinging jet at $\operatorname{Re}=2500$ and $H / d$ $=8$. 
(a)

(b)

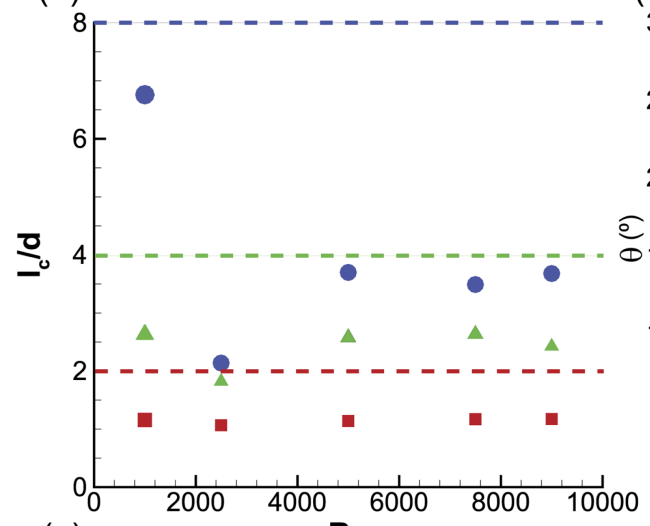

(c)

$\mathbf{R e}$
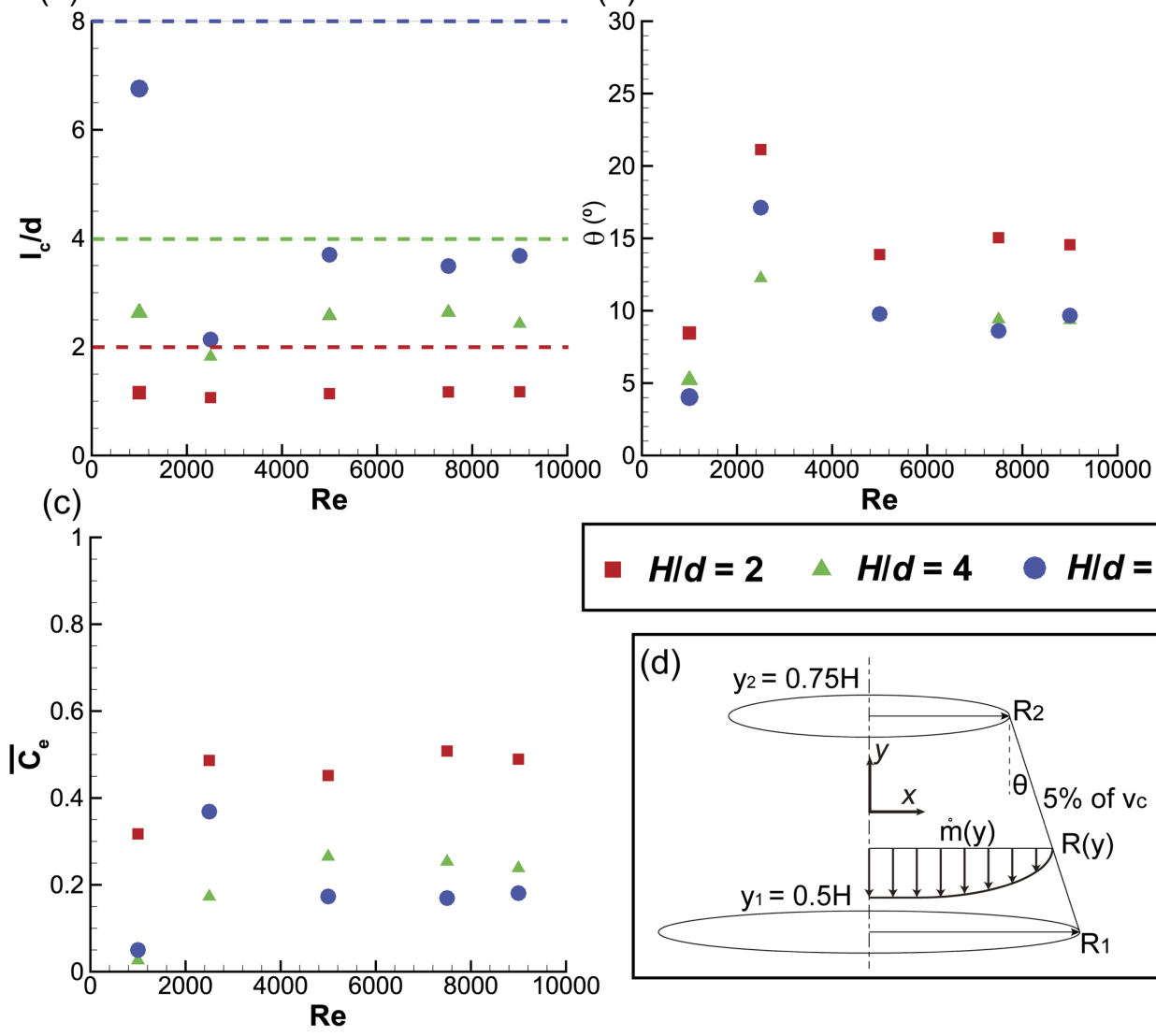

\section{- $H / d=2 \quad H / d=4 \quad \forall / d=8$}

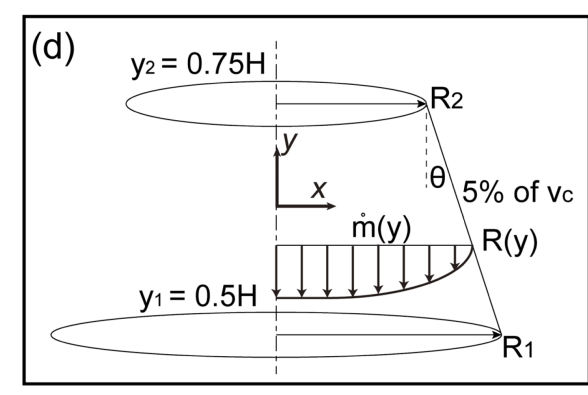

FIG. 6. The (a) jet core length non-dimensionalized by the jet orifice diameter, (b) jet expansion angle $(\theta)$, and (c) averaged mass entrainment coefficient $\left(\overline{C_{e}}\right)$ of the vertical jet as functions of the Reynolds number. Dashed lines in (a) indicate the positions of the bottom wall at each corresponding $H / d$ spacing. (d) shows a schematic diagram of the coefficient calculation.

one jet diameter) for the most confined case at $H / d=2$. This $H / d=2$ gap height also displays little variation in jet core length with increasing Reynolds number. The constant jet core length for $H / d=2$ implies that the highly confined geometry has a larger influence on the vertical jet development, which has the potential to further influence the wall jet development downstream. Interestingly, the jet core is shortest at $R e=2500$, which may indicate that a transition occurs around $\mathrm{Re}=2500$.

\section{B. Impinging jet characteristics upstream of the wall jet formation}

Observations of the expansion angle from Fig. 6(b) also show evidence of a transition occurring around $\mathrm{Re}=2500$. The expansion angles for the jets at $H / d=4$ and $H / d=8$ at $\operatorname{Re}=1000\left(\sim 4^{\circ}-5^{\circ}\right)$ are close to results from the literature $\left(\sim 2^{\circ}-3^{\circ}\right)$ for fully developed laminar jets. ${ }^{36}$ As the Reynolds number increases beyond 5000, the expansion angle stabilizes at approximately $10^{\circ}$, which is closer to the $12^{\circ}$ prediction of the Tollmien solution based on Prandtl's hypothesis. ${ }^{37}$ The high values shown at $\mathrm{Re}=2500$ for all confinement gap heights could be attributed to the fact that the vertical jet is undergoing a laminar-to-turbulent transition at or near this Reynolds number. The expansion angles at $H / d=2$ are consistently about $50 \%$ greater than the values of the less confined cases for all the Reynolds numbers investigated, indicating that the increased confinement is augmenting entrainment and thus causing the vertical jet to expand at a much greater angle before the impingement region.

The entrainment efficiency of the vertical jet was quantified with the averaged mass entrainment coefficient $\left(\overline{C_{e}}\right)$, similar to the approach proposed by Han and Mungal. ${ }^{38}$ As illustrated in the diagram in Fig. 6(d), the mass flow rate within the jet cone was obtained by integration according to $\dot{m}(y)$

$=\int_{0}^{R(y)} \rho v(x, y) 2 \pi x d x$, where $R(y)$ is the jet cone radius at height $y$. The dimensionless entrainment coefficient, $C_{e}(y)=\frac{d}{\dot{m}_{0}} \frac{d \dot{m}}{d y}$, was then calculated using a vertical range that is downstream of the orifice exit but also upstream of the impingement region (from $y=0.2 H$ to $0.9 H$, coinciding with the region used to determine the jet expansion angle). The mean entrainment coefficients within this height range at all the $H / d$ values and Reynolds numbers are plotted in Fig. 6(c). The higher entrainment coefficients at $H / d=2$ for all the Reynolds numbers investigated are likely caused by the relatively stable jet core length and larger expansion angle at this gap height. The small gap height of $H / d=2$ thus represents a more efficient geometry for entraining the surrounding liquid compared to the larger gap heights.

The plateauing of the three quantities shown in Fig. 6 at Reynolds numbers above 5000 implies a fully developed regime after a transition has occurred. To further investigate the turbulence characteristics of the impinging jets around this transition, the turbulent kinetic energy (TKE) is 


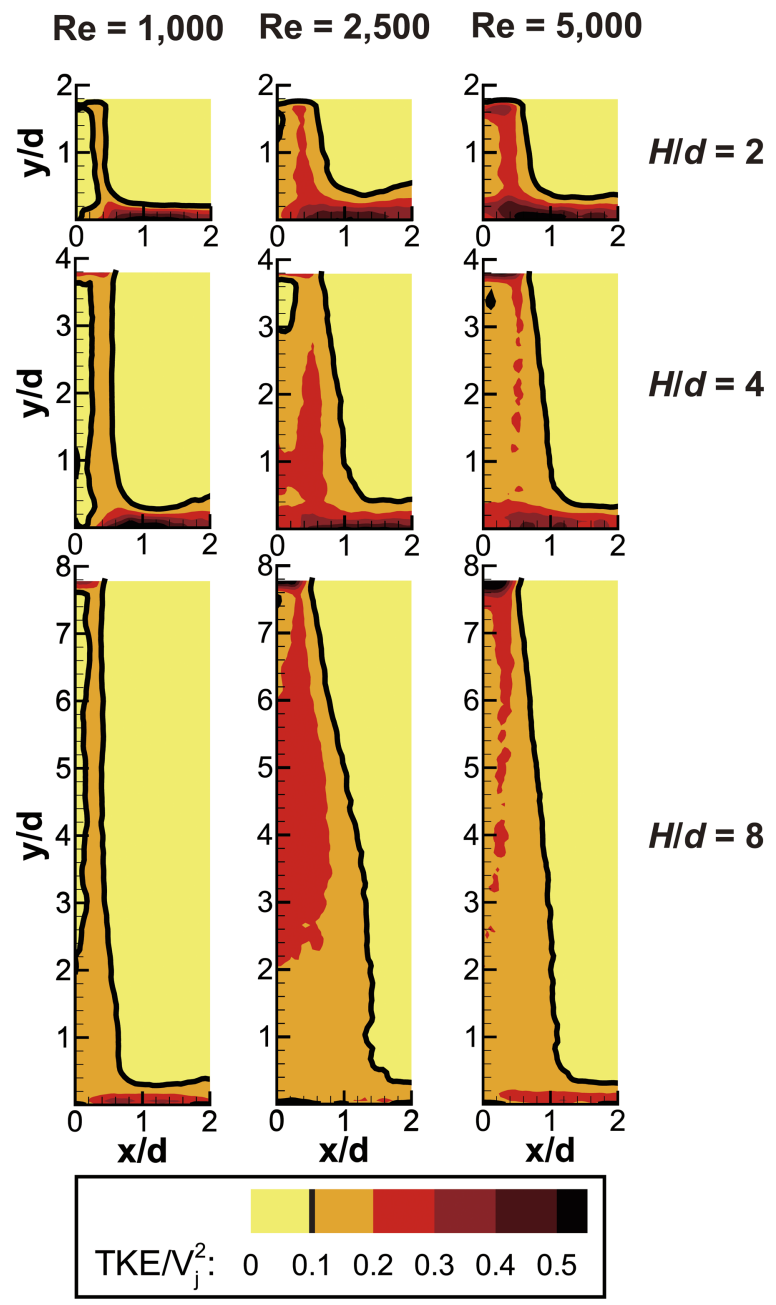

FIG. 7. Dimensionless turbulence kinetic energy (TKE) contours for $\operatorname{Re}=1000,2500$, and 5000 at $H / d=2,4$, and 8 . The contour line corresponding to $\mathrm{TKE} / V_{j}^{2}=10 \%$ is highlighted by bold lines.

calculated. TKE represents the mean kinetic energy per unit mass in the fluctuating velocity field and is calculated using $u^{\prime}, v^{\prime}$, and $w^{\prime}$ and non-dimensionalized by the square of the jet velocity $\left(V_{j}^{2}\right)$ at $\operatorname{Re}=1000,2500$, and 5000 for the three confinement heights. The contours of TKE are plotted in Fig. 7. For $\mathrm{Re}=1000$, TKE is only significant in the thin shear layer surrounding the vertical jet and the wall layer after impingement. At this low Reynolds number, TKE along the centerline of the jet remains low (with values less than 0.1), with the only exception of slightly higher turbulence values at the jet centerline for $H / d=8$ near the impingement surface at $y / d \approx 2.2$. This low turbulence intensity at the center of the jet suggests that the extended jet core at this Reynolds number is the result of an extended laminar potential region, which would preserve vertical jet momentum before impingement. In contrast, centerline turbulence is much higher for the $\mathrm{Re}=2500$ and 5000 cases, suggesting reduced impingement momentum. As shown in Fig. 6(a), except for the almost constant jet core lengths at $H / d=2$, the reduced jet core lengths at high Re compared with $\mathrm{Re}=1000$ for $H / d=4$ and 8 may also be attributed to the increased TKE level. Thus, the initial conditions for the developing wall jet at the higher Reynolds numbers have higher levels of turbulence and reduced momentum compared to the $R e=1000$ case. Circular free jets below $R e=1000$ are usually considered laminar, while jets with Reynolds numbers above 3000 are commonly considered as turbulent. ${ }^{39,40} \mathrm{~A}$ similar laminar-to-turbulent transition might explain the high TKE and non-monotonic behavior observed in jet core length and jet expansion angle at $\operatorname{Re}=2500$.

\section{Decay rate of the wall jet maximum velocity $\left(u_{m}\right)$}

To investigate the decay of the radial momentum in the wall jet region, the maximum of the radial-velocity component $\left(u_{m}\right)$, as defined in Fig. 1(b), is plotted at each $x / d$ location for $\operatorname{Re}=1000-9000$ with a bi-logarithmic scale in Fig. 8 for the confinement gap heights of $H / d=2,4$, and 8 . For $H / d=8$ (Fig. 8(c)), the linear decay of $u_{m}$ begins at $x / d=2.7$ and continues beyond $x / d=8$, out of the measurement domain. A similar region of linear decay spans from $x / d=2.1$ past $x / d=8$ for $H / d=4$ (Fig. 8(b)). In contrast, the region of linear decay of $u_{m}$ for $H / d=2$ (Fig. 8(a)) ends prior to $x / d=8$. The end of this linear region moves further downstream from the jet centerline as Reynolds number increases, e.g., ending at (a)

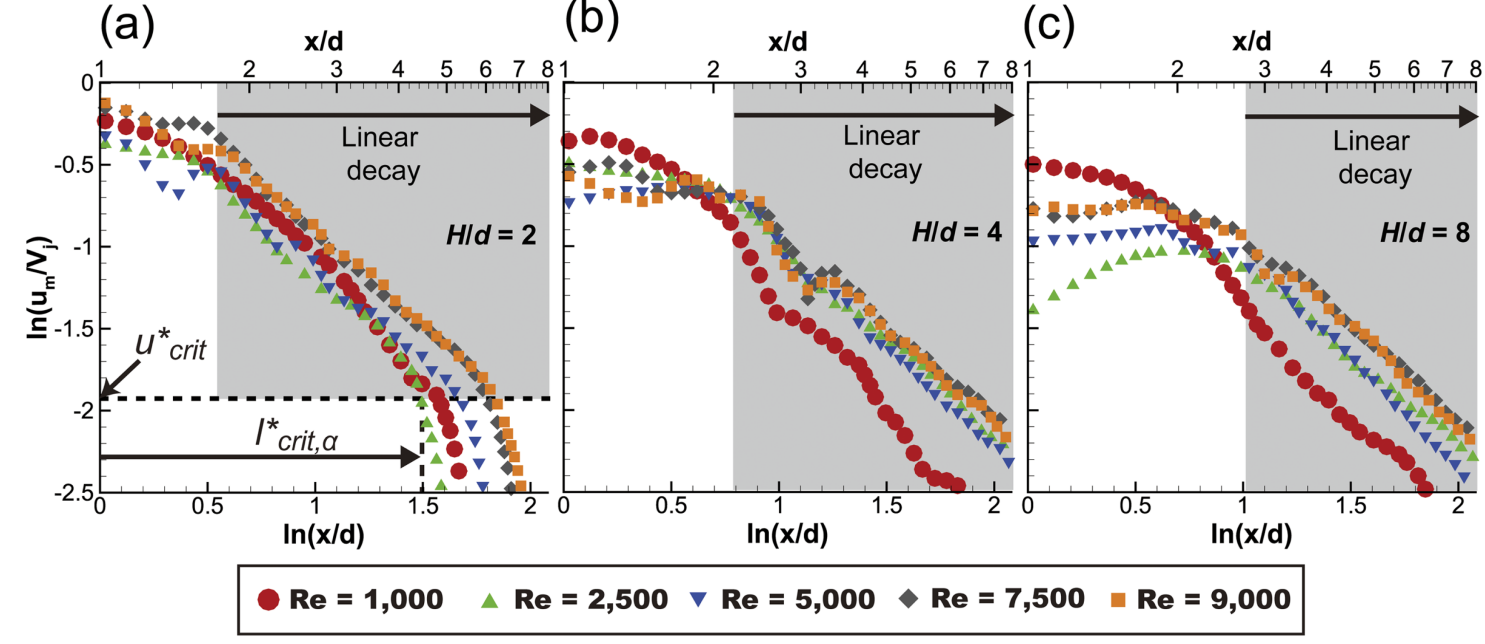

FIG. 8. Decay of $u_{m}$ for the confinement heights of (a) $H / d=2$, (b) $H / d=4$, and (c) $H / d=8$ in the range from $x / d=1$ to $x / d=8$. The shaded areas indicate the linear decay range. The dashed lines in (a) indicating $u_{c r i t}^{*}$ and $l_{c r i t, \alpha}^{*}$ for $\operatorname{Re}=2500$ are shown as an example. 


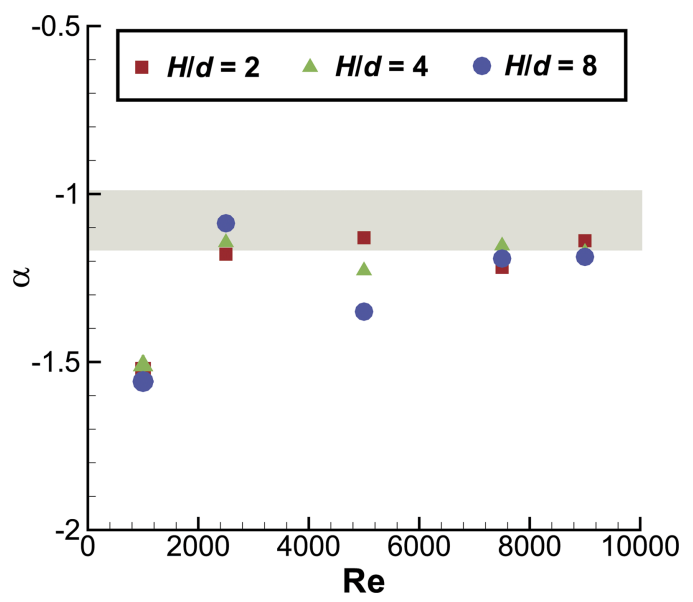

FIG. 9. Decay rate of $u_{m}$ as a function of both Reynolds number and confinement height $(H / d)$. The shaded area indicates the range of values from the literature listed in Table II.

$x / d=4.5$ for $\operatorname{Re}=2500$ versus $x / d=6$ for $\operatorname{Re}=9000$, indicating a dependence on the jet momentum. For the most confined cases $(H / d=2)$, a critical distance for the decay rate $\left(l_{c r i t}^{*}, \alpha\right)$ is defined as the dimensionless length from the jet centerline to the cessation location of the linear decay region of $u_{m}$. The critical velocity $\left(u_{c r i t}^{*}\right)$ is defined as the corresponding $u_{m}$ for each Reynolds number at $l_{c r i t, \alpha}^{*}$, normalized by the jet velocity $\left(V_{j}\right)$. Interestingly, while $l_{c r i t, \alpha}^{*}$ changes with jet Reynolds number, $u_{c r i t}^{*}$ for all Reynolds numbers is the same and equals to $u_{m} / V_{j}=0.15$.

A velocity decay rate is calculated by fitting an equation of the form of Equation (1) to the linear regions shown in Fig. 8. The resulting decay rate $(\alpha)$ for each case is plotted in Fig. 9. Representative results from the literature ${ }^{41-45}$ for radial wall jets are tabulated in Table II; for comparison, the range of values observed in these studies is denoted by the shaded area in Fig. 9. The values for $\alpha$ at $\operatorname{Re}=1000$ significantly differ from the literature, indicating a faster decay of wall jet velocity at this low Reynolds number. The decay rates for the higher Reynolds numbers $(\operatorname{Re}=2500,5000,7500$, and 9000$)$ are close to previous studies over a wide parameter range, including jets with and without confinement, nozzle-to-plate distances ranging from 0.5 to 25 , and Reynolds numbers ranging from 2500 to 288000 .

\section{Growth rate of the wall jet thickness $Y_{1 / 2, \mathrm{i}}$}

$Y_{1 / 2}$ for the top and wall layers for different confinement heights are plotted against $x / d$ locations using a bi-logarithmic scale at $\mathrm{Re}=1000$ in Fig. 10. A spline interpolation of the velocity profile is used to find the $y / d$ position where $u / u_{m}$ $=1 / 2$ occurs at each $x / d$ location, i.e., the values of $Y_{1 / 2, T}$ and $Y_{1 / 2, W}$. The wall jet scaling analysis is performed where the wall jet growth is linear, which is determined as the range of data where the growth of the top layer, growth of the wall layer, and decay of $u_{m}$ (as shown in Fig. 8) are all linear. The resulting ranges used for linear fitting and scaling analysis are identified with the solid lines overlaid on the plotted data in Fig. 10. The $x / d$ range where the linear growth occurs moves further downstream as the confinement height increases. For the most confined case $(H / d=2)$, a critical distance for the growth rate $\left(l_{\text {crit }}^{*}, \beta\right)$ is defined as the dimensionless length from the jet centerline to the cessation location of the linear growth region of $Y_{1 / 2}$ and is found to correlate with $l_{\text {crit }}^{*}, \alpha$.

The growth rates $(\beta)$ of $Y_{1 / 2}$ for top and wall layers resulting from the fit of Equation (2) to the data shown in Fig. 10 are listed in Table III. As the confinement height is reduced, the growth rate of the wall jet increases from $\beta_{T}=0.889$ at $H / d$ $=8$ to $\beta_{T}=1.340$ at $H / d=4$ and slightly drops to $\beta_{T}=1.275$ at $H / d=2$. The growth rate of the wall layer also increases from $\beta_{W}=0.868$ at $H / d=8$ to $\beta_{W}=1.347$ at $H / d=4$ and continues to increase to $\beta_{W}=1.746$ at $H / d=2$. The results show that the growth of the wall jet thickness accelerates as the confinement height is reduced for the current cases.

Values for $\beta_{i}$ from the literature ${ }^{10,41-44,46}$ are also tabulated in Table III and plotted together in Fig. 11 for comparison. Due to heater edge disturbance, the analysis for $Y_{1 / 2, W}$ in the wall layer at Re above 1000 was prevented. The current results show that for $\operatorname{Re}=1000$ at $H / d=4$ and 8 , there is little difference between $\beta_{i}$ for the top layer and wall layer. This contradicts the incomplete self-similarity proposed by Barenblatt et al. ${ }^{10}$ which postulates that the top layer and wall layer have different growth rates and thus do not achieve selfsimilarity. However, a large difference is observed for $\beta_{i}$ in the top and wall layers at $H / d=2$, indicating that incomplete self-similarity occurs at this confinement height. Results at additional confinement gap heights and Reynolds numbers are needed to verify whether the self-similarity shown at $H / d=$ 4 and $H / d=8$ is universally maintained at higher confinement gap heights and longer $x / d$ ranges.

The color circles in Fig. 11 represent the representative literature results for the growth rates of both planar and radial un-confined wall jets. For the previous studies, all the circles lie within the shaded area and have lower values of $\beta$. On the contrary, the black symbols correspond to the results for the current study which show higher values of $\beta$ for the overlapped Re range from 5000 to 10000 , indicating that the presence of the upper confinement plate accelerates the wall jet growth rates.

TABLE II. Decay rate of $u_{m}$ for radial wall jets from the literature.

\begin{tabular}{lccccc}
\hline \hline & $H / d$ & $x / d$ range & $\operatorname{Re}$ & $\alpha$ & Confinement \\
\hline Bakke (1957) & 0.5282 & $5-10$ & 3500 & -1.12 & No \\
Poreh et al. (1967) & $6.6,9.85,19.7$ & $10-60$ & $107000 \sim 288000$ & -1.1 & No \\
Tanaka and Tanaka (1977) & N/A & $2-100$ & $7500 \sim 55000$ & -1.09 & No \\
Knowles and Myszko (1998) & $2,4,8,10$ & $1-10$ & 90000 & -1.168 & No \\
Loureiro and Freire (2012) & 2 & $1-5$ & 47000 & -0.989 & Yes \\
\hline \hline
\end{tabular}




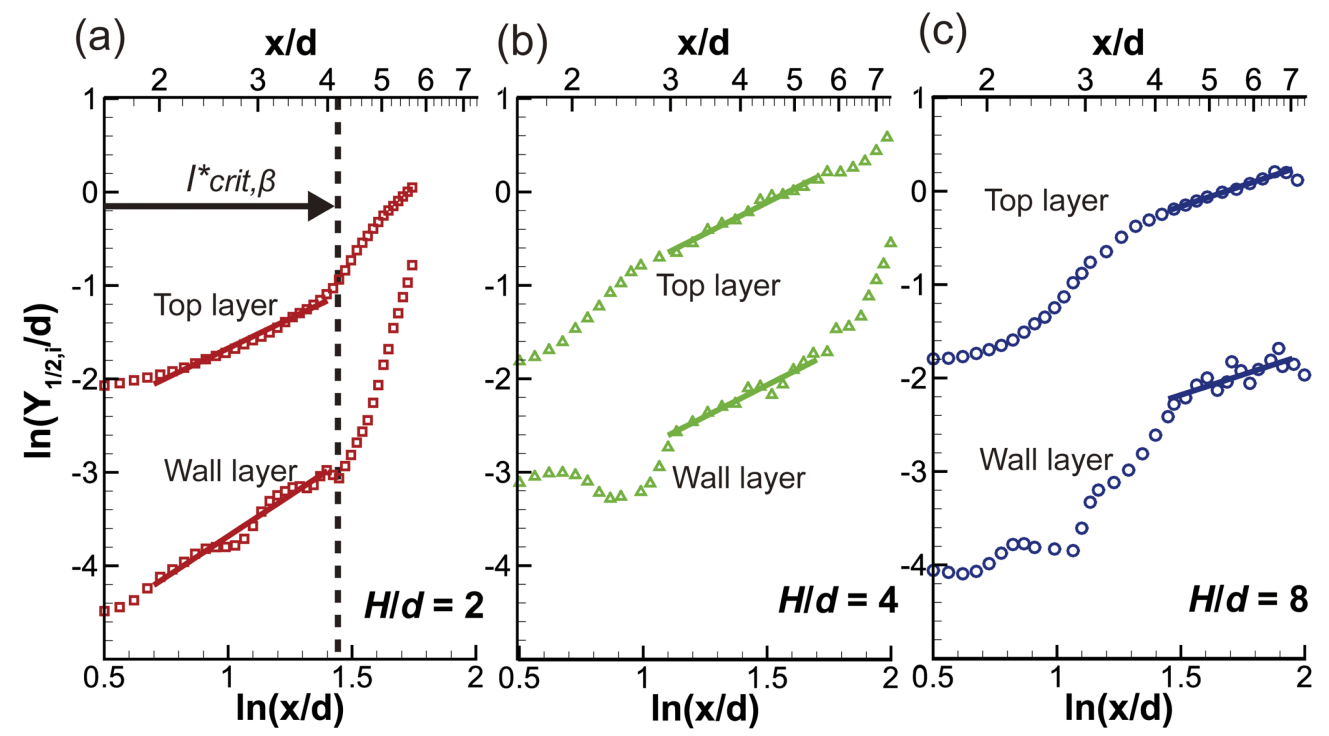

FIG. 10. $Y_{1 / 2, T}$ and $Y_{1 / 2, W}$ for (a) $H / d=2$, (b) $H / d=4$, and (c) $H / d=8$ at $\mathrm{Re}=1000$. The solid lines indicate the linear growth range that is used for scaling analysis. The dashed line in (a) shows an example of the critical distances of the linear growth of wall jet half-widths for all $H / d=2$ cases.

\section{E. Self-similarity of the wall jet velocity profile at $\operatorname{Re}=1000$}

To further investigate the self-similarity, normalized velocity profiles are plotted in Fig. 12. The velocity magnitude is normalized by $u_{m}$ at each $x / d$ location and the vertical distance from the bottom wall is non-dimensionalized by the nominal $y_{1 / 2, i}$, obtained using the fitted coefficients $\beta_{i}$ for both the top layer and the wall layer in Equation (2). The rescaled velocity profiles, shown in Fig. 12, are plotted in the range

TABLE III. Growth rate for both top and wall layers for Re $=1000$ and other cases from the literature.

\begin{tabular}{lcccccc}
\hline \hline & Type & $x / d$ range & Surface & Re & $\beta_{T}$ & $\beta_{W}$ \\
\hline$H / d=2$ & Radial & $2.0-4.0$ & Smooth & 1000 & 1.275 & 1.746 \\
$H / d=4$ & Radial & $3.0-5.5$ & Smooth & 1000 & 1.340 & 1.347 \\
$H / d=8$ & Radial & $4.1-6.7$ & Smooth & 1000 & 0.889 & 0.868 \\
Bakke (1957) & Radial & $5-10$ & Smooth & 3500 & 0.94 & N/A \\
Poreh et al. $(1967)$ & Radial & $10-60$ & Smooth & $107000-288000$ & 0.9 & N/A \\
Tanaka and Tanaka (1977) & Radial & $2-100$ & Smooth & $7500-55000$ & 0.97 & N/A \\
Knowles and Myszko (1998) $)$ & Radial & $1-10$ & Smooth & 90000 & 1 & N/A \\
Barenblatt et al. $(2005)$ & Planar & $40-150$ & Smooth & 9600 & 0.93 & 0.68 \\
Tang et al. (2015) & Planar & $30-80$ & Smooth & 7500 & 0.780 & 0.504 \\
Tang et al. (2015) & Planar & $30-80$ & Rough & 7500 & 0.817 & 0.403 \\
\hline \hline
\end{tabular}

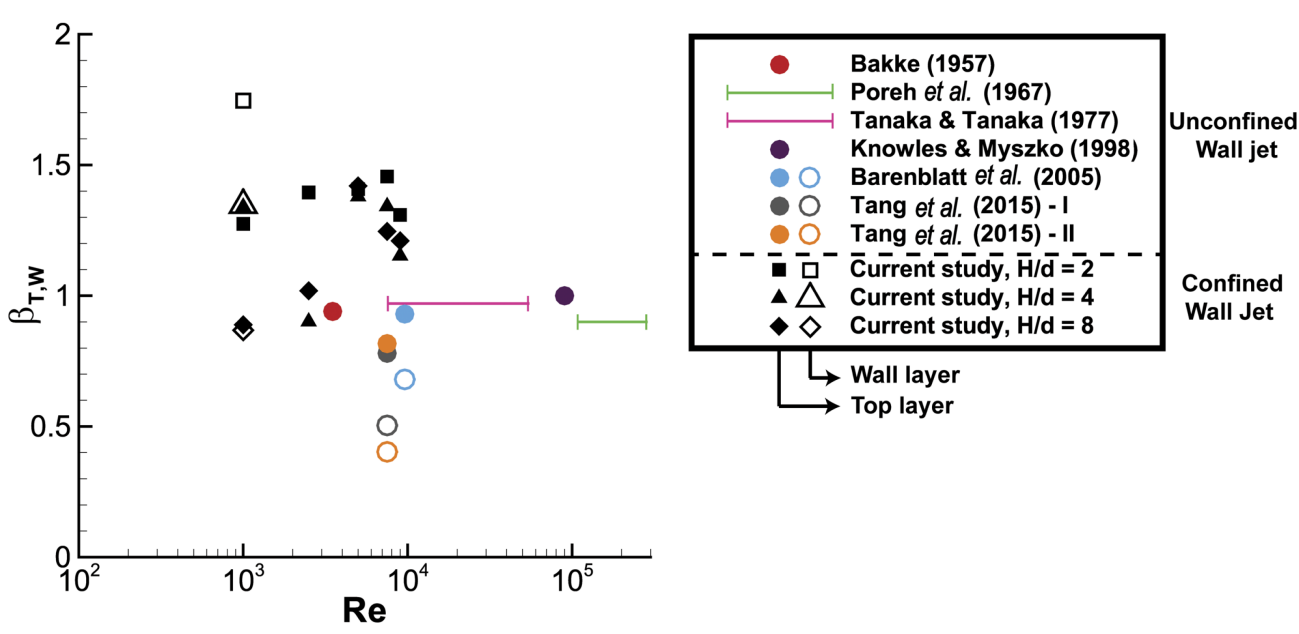

FIG. 11. Growth rate for both top and wall layers from the current study and the literature. Solid symbols show the results for the wall layer while the open symbols correspond to the top layer. The solid lines indicate a single value reported for the spanned Re range. 
(a)

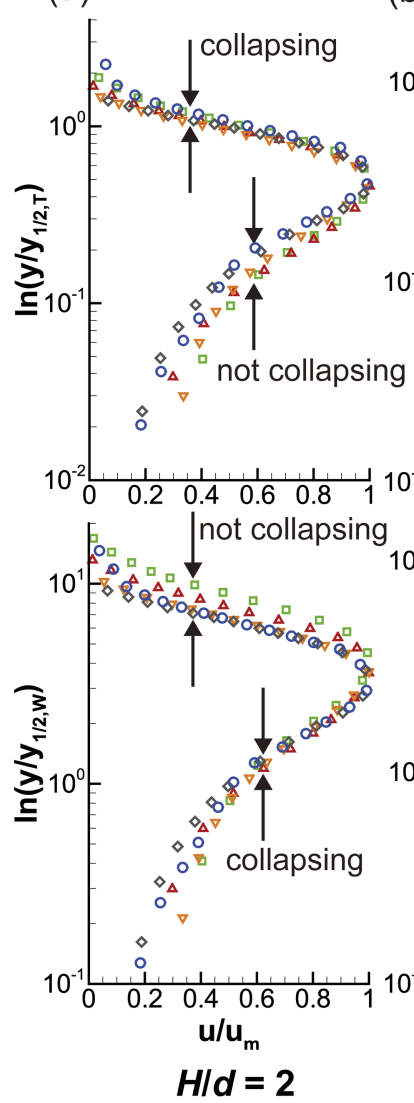

$2.1<x / d<4.1$ (b)

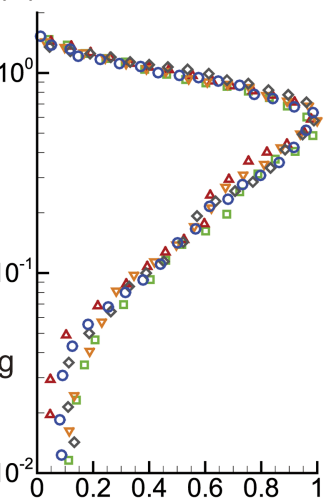

(c)
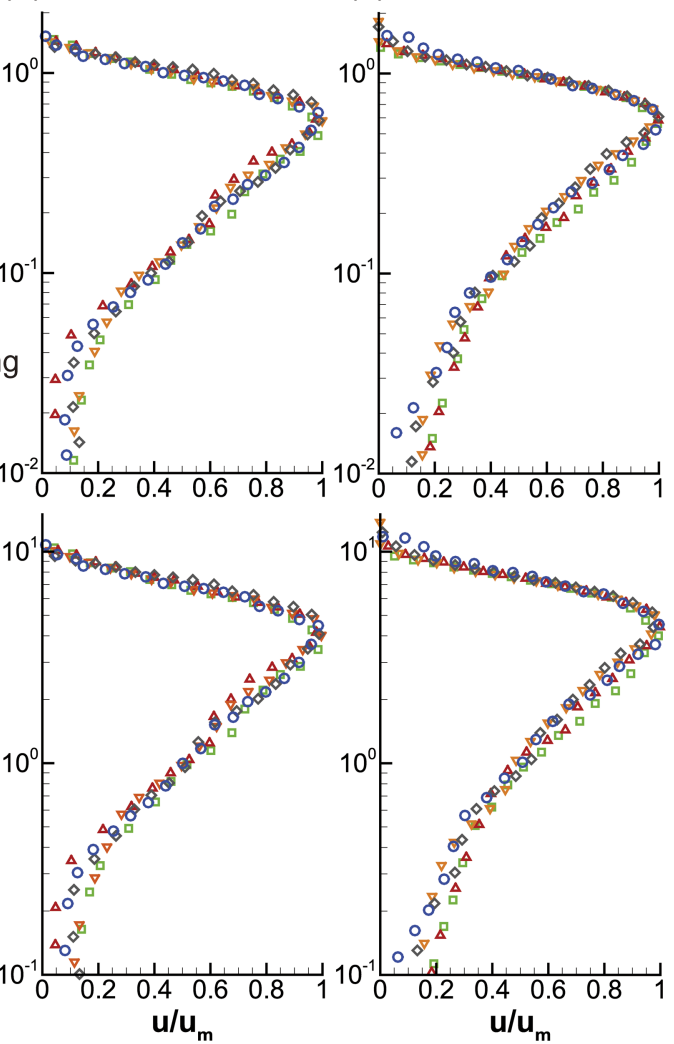

$H / d=4$

$3.1<x / d<5.0$

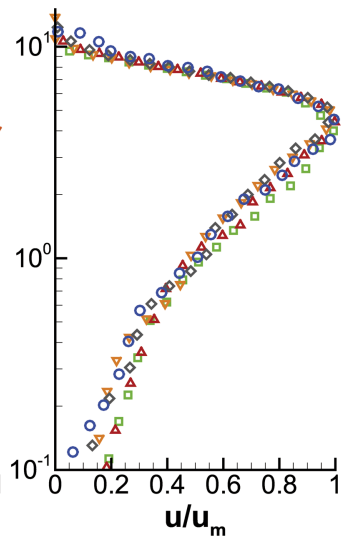

$H / d=8$ $4.5<x / d<6.5$
FIG. 12. Velocity profiles for $\operatorname{Re}=1000$ for (a) $H / d=2$, (b) $H / d=4$, and (c) $H / d=8$. Data in the top row are plotted with vertical distance $y$ non-dimensionalized by $y_{1 / 2, T}$, while data in the bottom row are plotted with vertical distance $y$ non-dimensionalized by $y_{1 / 2, W}$. Velocities are normalized by the $u_{m}$. where the linear growth of the wall jet holds, as discussed in Section III D.

As shown in Figs. 12(b) and 12(c), no major difference is observed between the upper and lower panels for $H / d$ $=4$ and $H / d=8$ where the velocity profiles are scaled with the top layer coefficients and wall layer coefficients, respectively. This result is expected given the small differences in $\beta_{T}$ and $\beta_{W}$ at these confinement gap heights. The velocity profiles for these cases show self-similarity that coincides with the linear growth range of $Y_{1 / 2, i}$, which contradicts the incomplete self-similarity proposed by Barenblatt et al. ${ }^{10}$ In contrast, the velocity profiles for $H / d=2$ shown in Fig. 12(a) display incomplete self-similarity. Only the top of the velocity profile collapses when non-dimensionalized with $\beta_{T}$, while only the wall layer of the profile collapses when non-dimensionalized with $\beta_{W}$.

\section{DISCUSSION}

Much of the information in the literature on wall jets has been obtained through the study of planar wall jets. ${ }^{47}$ The evolution of the length scales of planar and radial wall jets (i.e., the growth rate of the $Y_{1 / 2, i}$ ) has been shown to be very similar. ${ }^{48}$ Typical growth rates for both planar and radial wall jets are $\beta_{T} \approx 0.8-1 .^{10,41-44,46}$ However, the evolution of the velocity scales (i.e., the decay rate of $u_{m}$ ) is dependent on the wall jet geometry; the radial wall jet decelerates faster due to spreading in the azimuthal direction. Typical decay rates for planar wall jets range from $\alpha \approx-0.6$ to $-0.5,{ }^{10,46}$ which is much lower than the typical values for radial wall jets of $\alpha$ $\approx-1.1 .^{41,42}$ Thus, the decay rate of the maximum velocity in this study was only compared to results from radial wall jets in the literature, while results from both planar and radial studies were used for the comparison of growth rate in Section III D. To the authors' knowledge, the triple-layered structure of radial wall jets has not been investigated and the comparison was limited to planar wall jet studies.

The growth rate of the wall jet is found to be much higher at $H / d=2$ than at the larger confinement gap heights. At $H / d=8$, the $\beta_{T}$ and $\beta_{W}$ values are 0.889 and 0.868 , which are close to the values reported in the literature for unconfined wall jets. As the upper confinement plates move closer to the bottom wall, the values of $\beta_{T}$ and $\beta_{W}$ increase to 1.340 and 1.347 at $H / d=4$, and 1.275 and 1.746 at $H / d=$ 2. This trend indicates that smaller confinement gap heights increase the growth of the boundary layer on the bottom plate.

Confinement is not shown to have a large effect on the decay rate of $u_{m}$ in the current study. For Reynolds numbers above 2500 , the decay rate has a universal value $\alpha \approx-1.1$, regardless of confinement gap height and whether the velocity profile has reached self-similarity or not. Given these results, it is apparent that confinement does not have a strong influence on the decay rate of $u_{m}$, but it does alter self-similarity and the range where the linear growth occurs, as shown in Fig. 10. 


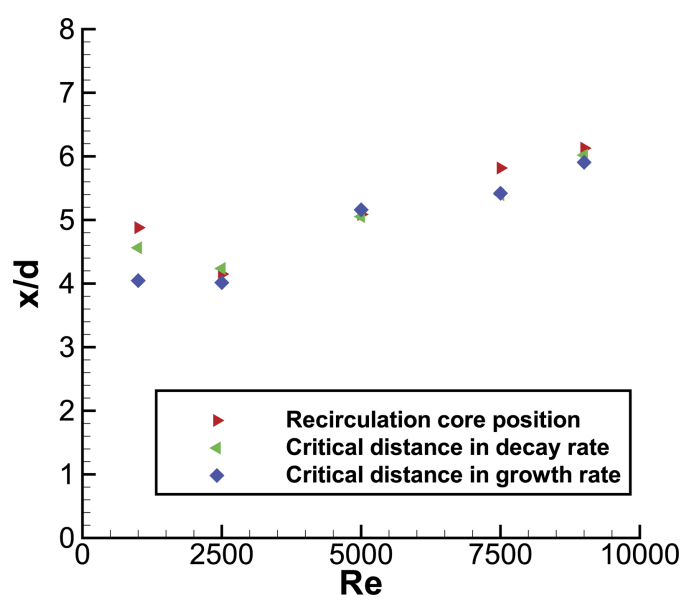

FIG. 13. The radial positions of the recirculation cores plotted with the critical distance in the linear decay of $u_{m}$ and critical distance in the linear growth of $Y_{1 / 2, i}$ for $H / d=2$ as a function of Reynolds number.

The hypothesis for the fast decay of $u_{m}$ at $\operatorname{Re}=1000$ is due to the unique shape of the velocity profile, as shown in Fig. 3(d). For Re above 5000 cases, the velocity profile is skewed towards the wall due to the lower position of $u_{m}$ than $\operatorname{Re}=1000$ cases. For $\operatorname{Re}=1000$, the profile is symmetric with respect to $u_{m}$, which is located farther away from the wall. Thus, the radial momentum associated with the $u$ component is easier to transfer to the surrounding liquid above the wall jet region, resulting in much faster decay of $u_{m}$. For Re above 5000, the momentum is more concentrated in a thin layer very next to the bottom wall, thus preserved better.

The critical distances for the decay rate $\left(l_{c r i t, \alpha}^{*}\right.$ in Fig. 8(a)) and growth rate $\left(l_{\text {crit }, \beta}^{*}\right.$ in Fig. 10(a)) indicate where the end of the linear decay of $u_{m}$ and linear growth of $Y_{1 / 2, i}$, respectively, occur at $H / d=2$. These critical distances as a function of Reynolds number are plotted in Fig. 13. The radial positions of the recirculation cores plotted with the critical distance in the linear decay of $u_{m}$ and critical distance in the linear growth of $Y_{1 / 2, i}$ for $H / d=2$ as a function of Reynolds number together with the recirculation core positions obtained from the centroids of the vortices, as shown in Fig. 4. For a given Reynolds number, the radial position of the recirculation core and the critical distances for growth rate and decay rate lie within one jet diameter of each other. This suggests that the early termination of the linear decay and growth region is caused by the recirculation of liquid within the gap at $H / d=2$. Boundary layer separation is caused by the axisymmetric vortex ring, which interrupts the development of the wall jet. The wall jet velocity decays exponentially until a critical value of maximum radial velocity $\left(u_{c r i t}^{*}\right)$ is reached, after which the radial momentum is weak enough to be overcome by the recirculation. The critical velocity is found to be $u_{c r i t}^{*} / V_{j}=0.15$ for all $H / d=2$ cases studied.

One important physical feature of the current experimental setup is that the orifice-to-plate distance is equal to the confinement height. This geometry imposes a no-slip condition on the entrained fluid nearest the orifice exit, which can affect the impinging jet stability and mixing characteristics with ambient liquid. ${ }^{49}$ Future studies may benefit from the use of a protruding nozzle with a fixed orifice-to-plate distance to further clarify the influence of confinement on the wall jet in confined jet impingement.

\section{CONCLUSIONS}

An experimental study using stereo particle image velocimetry to study the development of a confined radial wall jet is presented. The wall jet is formed downstream of a confined impinging jet, and the effect of the upper confinement plate on the wall jet development is studied. Results at three confinement gap heights $(H / d=2,4$, and 8) and five Reynolds numbers $(\operatorname{Re}=1000,2500,5000,7500$, and 9000) are presented. Confinement is found to alter the overall flow pattern in the confinement gap, which influences the wall jet development. Our finding of the loss of linear behavior only applies to wall jets formed by confined jet impingement.

Higher levels of turbulence in the vertical jet indicate that a transition occurs near $\mathrm{Re}=2500$. At Reynolds numbers above this transition, the decay rate of the maximum velocity in the developing region of the wall jet is found to have a universal constant close to -1.1 . This value is similar to the decay rate reported by other researchers for the fully developed region in radial wall jets. Confinement is found to not affect the velocity decay rate. At a Reynolds number of 1000 , the decay rate of maximum velocity is larger in magnitude than at the higher Reynolds numbers. The observed decay rate of -1.5 at this low Re demands further investigation but may be attributed to the different characteristics of the impinging vertical jet at this Reynolds number, including the jet core length, the jet expansion angle, and the mass entrainment coefficient.

No major difference has been found regarding the rate of growth for the top and wall layers of the wall jet at $\operatorname{Re}=1000$ for the $H / d=4$ and 8 confinement heights, which disagrees with the incomplete self-similarity proposed by Barenblatt et al. ${ }^{10}$ At a confinement gap height of $H / d$ $=2$, incomplete self-similarity is observed as the velocity profiles collapse with different top and wall layer scaling constants.

The counter-clockwise vortex structure observed at $H / d=2$ is found to interrupt the wall jet development at the smallest confinement gap height. The location of the recirculation coincides with the end of the linear wall jet development region. This result illustrates that the recirculation has a large influence on the wall jet development, as the boundary layer separation caused by this recirculation disrupts further development downstream.

\section{ACKNOWLEDGMENTS}

We would like to thank Dr. J. Weibel for the constructive comments and suggestions in the process of this manuscript. In addition, funding provided by National Science Foundation (NSF), PoLS-1205642; National Science Foundation, CBET-1336038; and National Science Foundation (NSF), IDBR-1152304. 
${ }^{1}$ J.-C. Han, "Recent studies in turbine blade cooling," Int. J. Rotating Mach. 10, 443-457 (2004).

${ }^{2}$ A. Sarkar, N. Nitin, M. V. Karwe, and R. P. Singh, "Fluid flow and heat transfer in air jet impingement in food processing," J. Food Sci. 69, 113-122 (2004).

${ }^{3}$ J. Ferrari, N. Lior, and J. Slycke, "An evaluation of gas quenching of steel rings by multiple-jet impingement," J. Mater. Process. Technol. 136, 190201 (2003).

${ }^{4}$ A. Pavlova and M. Amitay, "Electronic cooling using synthetic jet impingement," J. Heat Transfer 128, 897-907 (2006).

${ }^{5}$ D. T. H. New and S. C. M. Yu, Vortex Rings and Jets (Springer, 2015).

${ }^{6}$ S. V. Garimella, "Heat transfer and flow fields in confined jet impingement," Annu. Rev. Heat Transfer 11, 413 (2000).

${ }^{7}$ G. M. Carlomagno and A. Ianiro, "Thermo-fluid-dynamics of submerged jets impinging at short nozzle-to-plate distance: A review," Exp. Therm. Fluid Sci. 58, 15-35 (2014).

${ }^{8}$ B. R. Hollworth and S. I. Wilson, "Entrapment effects on impingement heat transfer: Part I-Measurements of heated jet velocity and temperature distributions and recovery temperatures on target surface," J. Heat Transfer 106, 797-803 (1984).

${ }^{9}$ M. B. Glauert, “The wall jet,” J. Fluid Mech. 1, 625-643 (1956).

${ }^{10}$ G. I. Barenblatt, A. J. Chorin, and V. M. Prostokishin, "The turbulent wall jet: A triple-layered structure and incomplete similarity," Proc. Natl. Acad. Sci. U. S. A. 102, 8850-8853 (2005).

${ }^{11}$ J. A. Fitzgerald and S. V. Garimella, "Flow field effects on heat transfer in confined jet impingement," J. Heat Transfer 119, 630-632 (1997).

${ }^{12} \mathrm{~J}$. A. Fitzgerald and S. V. Garimella, "A study of the flow field of a confined and submerged impinging jet,” Int. J. Heat Mass Transfer 41, 1025-1034 (1998).

${ }^{13} \mathrm{~S}$. V. Garimella and R. A. Rice, "Confined and submerged liquid jet impingement heat transfer," J. Heat Transfer 117, 871-877 (1995).

${ }^{14}$ M. J. Rau and S. V. Garimella, "Local two-phase heat transfer from arrays of confined and submerged impinging jets," Int. J. Heat Mass Transfer 67, 487-498 (2013).

${ }^{15}$ M. J. Rau, T. Guo, P. P. Vlachos, and S. V. Garimella, "Stereo-PIV measurements of vapor-induced flow modifications in confined jet impingement boiling,” Int. J. Multiphase Flow 84, 19-33 (2016).

${ }^{16}$ M. J. Rau, P. P. Vlachos, and S. V. Garimella, "A tomographic-PIV investigation of vapor-induced flow structures in confined jet impingement boiling," Int. J. Multiphase Flow 84, 86-97 (2016).

${ }^{17} \mathrm{C}$. Willert, "Stereoscopic digital particle image velocimetry for application in wind tunnel flows," Meas. Sci. Technol. 8, 1465-1479 (1997).

${ }^{18} \mathrm{~J}$. Westerweel, "Efficient detection of spurious vectors in particle image velocimetry data," Exp. Fluids 16-16, 263-272 (1994).

${ }^{19}$ Z. Xue, J. J. Charonko, and P. P. Vlachos, "Particle image pattern mutual information and uncertainty estimation for particle image velocimetry," Measurement Sci. Technol. 26(7), 074001 (2015).

${ }^{20}$ A. K. Prasad, "Stereoscopic particle image velocimetry," Exp. Fluids 29, 103-116 (2000).

${ }^{21}$ B. Wieneke, "Stereo-PIV using self-calibration on particle images," Exp. Fluids 39, 267-280 (2005).

${ }^{22}$ S. M. Soloff, R. J. Adrian, and Z.-C. Liu, "Distortion compensation for generalized stereoscopic particle image velocimetry," Meas. Sci. Technol. 8, 1441-1454 (1999).

${ }^{23}$ A. Eckstein, J. Charonko, and P. P. Vlachos, "Phase correlation processing for DPIV measurements," Exp. Fluids 45, 485-500 (2008).

${ }^{24}$ A. Eckstein and P. P. Vlachos, "Digital particle image velocimetry (DPIV) robust phase correlation," Meas. Sci. Technol. 20, 55401 (2009).

${ }^{25}$ A. Eckstein and P. P. Vlachos, "Assessment of advanced windowing techniques for digital particle image velocimetry (DPIV)," Meas. Sci. Technol. 20, 075402 (2009).
${ }^{26}$ E. Delnoij, J. Westerweel, N. G. Deen, J. a. M. Kuipers, and W. P. M. van Swaaij, "Ensemble correlation PIV applied to bubble plumes rising in a bubble column," Chem. Eng. Sci. 54, 5159-5171 (1999).

${ }^{27}$ C. J. Kähler, S. Scharnowski, and C. Cierpka, "On the resolution limit of digital particle image velocimetry,” Exp. Fluids 52, 1629-1639 (2012).

${ }^{28} \mathrm{~B}$. Wieneke and K. Pfeiffer, "Adaptive PIV with variable interrogation window size and shape," in the 15th International Symposium on Applications of Laser Techniques to Fluid Mechanics, Lisbon, Portugal, 5-8 July 2010, pp. 5-8.

${ }^{29}$ J. Westerweel, P. F. Geelhoed, and R. Lindken, "Single-pixel resolution ensemble correlation for micro-PIV applications," Exp. Fluids 37, 375-384 (2004).

${ }^{30}$ F. Scarano, "Iterative image deformation methods in PIV," Meas. Sci. Technol. 13, R1-R19 (2001).

${ }^{31}$ J. Westerweel and F. Scarano, "Universal outlier detection for PIV data," Exp. Fluids 39, 1096-1100 (2005).

${ }^{32}$ G. K. Morris and S. V. Garimella, "Orifice and impingement flow fields in confined jet impingement," Trans. ASME J. Electron. Packaging 120, 68-72 (1998).

${ }^{33}$ G. K. Morris, S. V. Garimella, and J. A. Fitzgerald, "Flow-field prediction in submerged and confined jet impingement using the Reynolds stress model," Trans. ASME J. Electron. Packaging 121(4), 255-262 (1998).

${ }^{34}$ J. Zhou, R. J. Adrian, S. Balachandar, and T. M. Kendall, "Mechanisms for generating coherent packets of hairpin vortices in channel flow," J. Fluid Mech. 387, 353-396 (1999).

${ }^{35} \mathrm{~S}$. Ashforth-Frost, K. Jambunathan, and C. F. Whitney, "Velocity and turbulence characteristics of a semiconfined orthogonally impinging slot jet," Exp. Therm. Fluid Sci. 14, 60-67 (1997).

${ }^{36}$ E. P. Symons and T. L. Labus, "Experimental investigation of an axisymmetric fully developed laminar free jet," NASA Technical Note D-6304, 1971.

${ }^{37}$ G. Horn and M. W. Thring, "Angle of spread of free jets," Nature 178, 205-206 (1956)

${ }^{38}$ D. Han and M. G. Mungal, "Direct measurement of entrainment in reacting/nonreacting turbulent jets," Combust. Flame 124, 370-386 (2001).

${ }^{39}$ J. Gauntner, J. Livingood, and P. Hrycak, "Survey of literature on flow characteristics of a single turbulent jet impinging on a flat plate," NASA Technical Note D-5652, 1970.

${ }^{40} \mathrm{~L}$. Boguslawski and C. O. Popiel, "Flow structure of the free round turbulent jet in the initial region," J. Fluid Mech. 90, 531-539 (1979).

${ }^{41}$ P. Bakke, "An experimental investigation of a wall jet," J. Fluid Mech. 2, 467-472 (1957).

${ }^{42}$ M. Poreh, Y. G. Tsuei, and J. E. Cermak, "Investigation of a turbulent radial wall jet," J. Appl. Mech. 34, 9-81 (1967).

${ }^{43}$ T. Tanaka and E. Tanaka, "Experimental studies of a radial turbulent jet: 2nd report, wall jet on a flat smooth plate," Bull. JSME 20(140), 209-215 (1977).

${ }^{44}$ K. Knowles and M. Myszko, "Turbulence measurements in radial wall-jets," Exp. Therm. Fluid Sci. 17, 71-78 (1998).

${ }^{45}$ J. B. R. Loureiro and A. P. S. Freire, "On the dynamic behaviour of turbulent impinging jets," in Proceedings of the Seventh International Symposium On Turbulence, Heat and Mass Transfer, Palermo, Italy, 24-27 September 2012, pp. $1-12$.

${ }^{46}$ Z. Tang, N. Rostamy, D. J. Bergstrom, J. D. Bugg, and D. Sumner, "Incomplete similarity of a plane turbulent wall jet on smooth and transitionally rough surfaces," J. Turbul. 16, 1076-1090 (2015).

${ }^{47}$ B. E. Launder and W. Rodi, "The turbulent wall jet measurements and modeling," Annu. Rev. Fluid Mech. 15, 429-459 (1983).

${ }^{48}$ R. Banyassady, "Large-eddy simulations of plane and radial wall-jets over smooth and rough surfaces," Ph.D Dissertation (Queen's University, 2015).

${ }^{49}$ J. Mi, G. J. Nathan, and D. S. Nobes, "Mixing characteristics of axisymmetric free jets from a contoured nozzle, an orifice plate and a pipe," J. Fluids Eng. 123, 878 (2001). 